\title{
Bosons in a Trap: A Rigorous Derivation of the Gross-Pitaevskii Energy Functional
}

\author{
Elliott H. Lieb ${ }^{1}$, Robert Seiringer ${ }^{2}$, and Jakob Yngvason ${ }^{2}$ \\ 1. Departments of Physics and Mathematics, Jadwin Hall, \\ Princeton University, P. O. Box 708, Princeton, New Jersey 08544 \\ 2. Institut für Theoretische Physik, Universität Wien \\ Boltzmanngasse 5, A 1090 Vienna, Austria
}

5 August, 1999

\begin{abstract}
The ground state properties of interacting Bose gases in external potentials, as considered in recent experiments, are usually described by means of the Gross-Pitaevskii energy functional. We present here the first proof of the asymptotic exactness of this approximation for the ground state energy and particle density of a dilute Bose gas with a positive interaction.
\end{abstract}

\section{Introduction}

Recent experimental breakthroughs in the treatment of dilute Bose gases have renewed interest in formulas for the ground state and its energy derived many decades ago. One of these is the Gross-Pitaevskii (GP) formula for the energy in a trap [1]-[3], such as is used in the actual experiments. We refer to

\footnotetext{
${ }^{1}$ Work partially supported by U.S. National Science Foundation grant PHY 98-20650. (C) 1999 by the authors. This paper may be reproduced, in its entirety, for non-commercial purposes.
} 
[4] for an up to date review of this approximation and its applications. One of the inputs needed for its justification is the ground state energy per unit volume of a dilute, thermodynamically infinite, homogeneous gas. This latter quantity has been known for many years, but it was only very recently that it was derived rigorously [5] for suitable interparticle potentials. Consequently, it is appropriate now to use this new result to go one step further and derive the GP formula rigorously.

The starting point for our investigation is the Hamiltonian for $N$ identical bosons

$$
H^{(N)}=\sum_{i=1}^{N}\left(-\nabla_{i}^{2}+V\left(x_{i}\right)\right)+\sum_{i<j} v\left(\left|x_{i}-x_{j}\right|\right)
$$

acting on totally symmetric, square integrable wave functions of $\left(x_{1}, \ldots, x_{N}\right)$ with $x_{i} \in \mathbb{R}^{3}$. Units have here been chosen so that $\hbar=2 m=1$, where $m$ is the mass. We consider external potentials $V$ that are measurable and locally bounded and tend to infinity for $|x| \rightarrow \infty$ in the sense that inf $|x| \geq R V(x) \rightarrow \infty$ for $R \rightarrow \infty$. The potential is then bounded below and for convenience we assume that its minimum value is zero. The ground state of $-\nabla^{2}+V(x)$ provides a natural energy unit, $\hbar \omega$, and the corresponding length unit, $\sqrt{\hbar / m \omega}$, describes the extension of the potential. We shall measure all energies and lengths in these units. In the available experiments $V$ is typically $\sim|x|^{2}$ and $\sqrt{\hbar / m \omega}$ of the order $10^{-6} \mathrm{~m}$.

The particle interaction $v$ is assumed to be positive, spherically symmetric and decay faster than $|x|^{-3}$ at infinity. In particular, the scattering length, denoted by $a$, should be finite. We recall that the (two-body) scattering length is defined by means of the solution $u(r)$ of the zero energy scattering equation

$$
-u^{\prime \prime}(r)+\frac{1}{2} v(r) u(r)=0
$$

with $u(0)=0$; by definition, $a=\lim _{r \rightarrow \infty}\left(r-u(r) / u^{\prime}(r)\right)$. Let $v_{1}(r)$ be a fixed potential with scattering length $a_{1}$. Then $v(r)=\left(a_{1} / a\right)^{2} v_{1}\left(a_{1} r / a\right)$ has scattering length $a$. We regard in the following $v_{1}$ as fixed, but vary $a$ (in fact, we shall take $\left.a=a_{1} / N\right)$. The ground state energy $E^{\mathrm{QM}}$ of (1.1) depends on the potentials $V$ and $v$, besides $N$, but with $V$ fixed and $v(r)=$ $\left(a_{1} / a\right)^{2} v_{1}\left(a_{1} r / a\right)$, the notation $E^{\mathrm{QM}}(N, a)$ is justified. The corresponding eigenfunction will be denoted $\Psi_{0}^{(N)}$. It is unique up to a phase that can be 
chosen such that the wave function is strictly positive where the interaction is finite [7]. The particle density is defined by

$$
\rho_{N, a}^{\mathrm{QM}}(x)=N \int_{\mathbb{R}^{3(N-1)}}\left|\Psi_{0}^{(N)}\left(x, x_{2}, \ldots, x_{N}\right)\right|^{2} d^{3} x_{2} \ldots d^{3} x_{N} .
$$

The Gross-Pitaevskii (GP) energy functional is defined as

$$
\mathcal{E}^{\mathrm{GP}}[\Phi]=\int_{\mathbb{R}^{3}}\left(|\nabla \Phi(x)|^{2}+V(x)|\Phi(x)|^{2}+4 \pi a|\Phi(x)|^{4}\right) d^{3} x
$$

where $\Phi$ is a function on $\mathbb{R}^{3}$. For a given $N$ the corresponding GP energy, denoted $E^{\mathrm{GP}}(N, a)$, is defined as the infimum of $\mathcal{E}[\Phi]$ under the normalization condition

$$
\int_{\mathbb{R}^{3}}|\Phi(x)|^{2} d^{3} x=N
$$

It has the simple scaling property

$$
E^{\mathrm{GP}}(N, a)=N E^{\mathrm{GP}}(1, N a) .
$$

What (1.6) shows is that the GP functional (1.4) together with the normalization condition (1.5) has one characteristic parameter, namely $\mathrm{Na}$. (Recall that lengths are measured in the unit $\sqrt{\hbar / m \omega}$ associated with $V$ so $a$ is dimensionless.) Thus, if we want to investigate the non-trivial aspects of GP theory we have to consider a limit in which $N \rightarrow \infty$ with $N a$ fixed. This explains the seemingly peculiar limit in Theorems 1.1 and 1.2. As $N a \rightarrow \infty$ the GP energy functional simplifies, since the gradient term becomes small compared to the other terms, and the so called "Thomas-Fermi limit" described in Theorem 2.2 results. In some typical experiments $a$ is about $10^{-3}$, while $N$ varies from $10^{3}$ to $10^{7}$. Thus $a_{1}$ in Theorems 1.1 and 1.2 varies from 1 to about $10^{4}$.

In the next section it will be shown that the infimum of the energy functional (1.4), under the subsidiary condition (1.5), is obtained for a unique, strictly positive function, denoted $\Phi^{\mathrm{GP}}$. The GP density is given by

$$
\rho_{N, a}^{\mathrm{GP}}(x)=\Phi^{\mathrm{GP}}(x)^{2} .
$$


It satisfies

$$
\rho_{N, a}^{\mathrm{GP}}(x)=N \rho_{1, N a}^{\mathrm{GP}}(x) .
$$

The main result of this paper concerns the behavior of the quantum mechanical ground state energy $E^{\mathrm{QM}}(N, a)$ when $N$ is large, but $a$ is small, so that $N a$ is $O(1)$. It is important to note that although the density tends to infinity for $N \rightarrow \infty$ (by Eq. (1.8)) we are still concerned with dilute systems in the sense that $a^{3} \bar{\rho} \ll 1$, where

$$
\bar{\rho}=\frac{1}{N} \int \rho_{N, a}^{\mathrm{GP}}(x)^{2} d^{3} x
$$

is the mean GP density. (Note the exponent 2 in (1.9).) In fact, since $a \sim N^{-1}, a^{3} \bar{\rho} \sim N^{-2}$.

The precise statement of the limit theorem for the energy is as follows.

Theorem 1.1 (The GP energy is the dilute limit of the QM energy). For every fixed $a_{1}$

$$
\lim _{N \rightarrow \infty} \frac{E^{\mathrm{QM}}\left(N, a_{1} / N\right)}{N}=E^{\mathrm{GP}}\left(1, a_{1}\right)
$$

and the convergence is uniform on bounded intervals of $a_{1}$.

While we do not prove anything about Bose-Einstein condensation, which necessarily involves the full one-body density matrix $\rho^{(1)}\left(x, x^{\prime}\right)$, we can make an assertion about the diagonal part of the density matrix, $\rho^{\mathrm{QM}}(x)=$ $\rho^{(1)}(x, x)$ :

Theorem 1.2 (The GP density is the dilute limit of the QM density). For every fixed $a_{1}$

$$
\lim _{N \rightarrow \infty} \frac{1}{N} \rho_{N, a_{1} / N}^{\mathrm{QM}}(x)=\rho_{1, a_{1}}^{\mathrm{GP}}(x)
$$

in the sense of weak convergence in $L^{1}$.

For the proof of Theorem 1.1 we establish upper and lower bounds on $E^{\mathrm{QM}}(N, a)$ in terms of $E^{\mathrm{GP}}(N, a)$ with controlled errors. Theorem 1.2 follows from Theorem 1.1 by variation of the external potential. The upper bound is obtained in Section 3 by a variational calculation which generalizes the upper 
bound of Dyson [6] for a homogeneous gas of hard spheres. We also derive an upper bound on the chemical potential, i.e., the energy increase when one particle is added to the system. This upper bound is used in the proof of the lower bound of the energy in Section 4. The main ingredient for the lower bound, however, is the bound for the homogeneous case established in [5]. In addition, some basic properties of the minimizer of the GP functional are used in the proof and we consider them next.

\section{The Gross-Pitaevskii Energy Functional}

The GP functional is defined by (1.4) for $\Phi \in \mathcal{D}$ with

$$
\mathcal{D}=\left\{\Phi: \nabla \Phi \in L^{2}\left(\mathbb{R}^{3}\right), V|\Phi|^{2} \in L^{1}\left(\mathbb{R}^{3}\right), \Phi \in L^{4}\left(\mathbb{R}^{3}\right) \cap L^{2}\left(\mathbb{R}^{3}\right)\right\},
$$

where $f \in L^{p}\left(\mathbb{R}^{n}\right)$ means $\int_{\mathbb{R}^{n}}|f(x)|^{p} d^{n} x<\infty$. The corresponding GP energy is given by

$$
E^{\mathrm{GP}}(N, a)=\inf \left\{\mathcal{E}^{\mathrm{GP}}[\Phi]: \Phi \in \mathcal{D}_{N}\right\}
$$

with

$$
\mathcal{D}_{N}=\mathcal{D} \cap\left\{\Phi: \int|\Phi(x)|^{2} d^{3} x=N\right\} .
$$

The basic facts about the GP functional are summarized in the following theorem.

Theorem 2.1 (Existence and properties of a minimizer). The infimum in (2.2) is a minimum, i.e., there is a $\Phi^{\mathrm{GP}} \in \mathcal{D}_{N}$ such that $E^{\mathrm{GP}}(N, a)=\mathcal{E}^{\mathrm{GP}}\left[\Phi^{\mathrm{GP}}\right]$. This $\Phi^{\mathrm{GP}}$ is unique up to a phase factor, which can be chosen so that $\Phi^{\mathrm{GP}}$ is strictly positive. $\Phi^{\mathrm{GP}}$ is at least once continuously differentiable, and if $V$ is $C^{\infty}$ then also $\Phi^{\mathrm{GP}}$ is $C^{\infty}$. The energy $E^{\mathrm{GP}}(N, a)$ is continuously differentiable in $a$ and hence (by Eq. (1.0)) also in $N$. The minimizer $\Phi^{\mathrm{GP}}$ solves the Gross-Pitaevskii equation

$$
-\nabla^{2} \Phi+V \Phi+8 \pi a|\Phi|^{2} \Phi=\mu \Phi
$$

(in the sense of distributions) with

$$
\mu=d E^{\mathrm{GP}}(N, a) / d N=E^{\mathrm{GP}}(N, a) / N+4 \pi a \bar{\rho} .
$$

Here $\bar{\rho}$ is the mean density (1.9). 
The GP energy functional is mathematically quite similar to the energy functional of Thomas-Fermi-von Weizsäcker theory and Theorem 2.1 can be proved by the methods of Sect. VII in [8]. For completeness, the proof is given in Appendix A. With additional properties of $V$ one can draw further conclusions about $\Phi^{\mathrm{GP}}$ :

Proposition 2.1 (Symmetry and monotonicity). If $V$ is spherically symmetric and monotone increasing, then $\Phi^{\mathrm{GP}}$ is spherically symmetric and monotone decreasing.

Proof. Let $\Phi^{*}$ be the symmetric-decreasing rearrangement of $\Phi^{\mathrm{GP}}$ (see [9]). Then $\mathcal{E}^{\mathrm{GP}}\left[\Phi^{*}\right] \leq \mathcal{E}^{\mathrm{GP}}\left[\Phi^{\mathrm{GP}}\right]$.

Proposition 2.2 ( $\mathrm{Log}$ concavity). If $V$ is convex, then $\Phi^{\mathrm{GP}}$ is $\log$ concave, i.e. $\Phi^{\mathrm{GP}}(x)^{\lambda} \Phi^{\mathrm{GP}}(y)^{(1-\lambda)} \leq \Phi^{\mathrm{GP}}(\lambda x+(1-\lambda) y)$, for all $x, y \in \mathbb{R}^{3}$, $\lambda \in(0,1)$.

Proof. Using the Trotter product formula it suffices to show that the solutions $u(t, x)$ of the equations

$$
\frac{\partial u}{\partial t}-\nabla^{2} u=0, \quad \frac{\partial u}{\partial t}+V u=0, \quad \frac{\partial u}{\partial t}+8 \pi a u^{3}=\mu u
$$

are $\log$ concave, if $u(0, x)$ is $\log$ concave. The first follows from the fact, that the convolution of two log concave functions is log concave, the second follows easily from convexity of $V$, and the third is shown in [11.

The GP theory has a well defined limit if $N a \rightarrow \infty$. It is sometimes referred to as the "Thomas-Fermi limit" of GP theory because the gradient term vanishes in this limit. For simplicity we restrict ourselves to homogeneous external potentials $V$, i.e.,

$$
V(\lambda x)=\lambda^{s} V(x)
$$

for some $s>0$.

Theorem 2.2 (Large $\mathbf{N a}$ limit). Let $V$ be homogeneous of order s and let $\mathcal{F}$ be the functional

$$
\mathcal{F}[\rho]=\int_{\mathbb{R}^{3}}\left(V(x) \rho(x)+4 \pi a \rho(x)^{2}\right) d^{3} x
$$


with $\rho(x) \geq 0, x \in \mathbb{R}^{3}$. Let $F(N, a)$ be the infimum of $\mathcal{F}$ under the condition $\int \rho=N$. By scaling, $F(N, a)=N F(1, N a)$ and $F(1, N a)=$ $(N a)^{s /(s+3)} F(1,1)$. In the limit $N a \rightarrow \infty$ we have

$$
\lim _{N a \rightarrow \infty} \frac{E^{\mathrm{GP}}(1, N a)}{(N a)^{s /(s+3)}}=F(1,1) .
$$

The minimizing density of $\mathcal{F}$ under the condition $\int \rho=1$ and with $a=1$ is given by

$$
\rho_{1,1}^{\mathrm{F}}(x)=(8 \pi)^{-1}[\widetilde{\mu}-V(x)]_{+}
$$

with $\widetilde{\mu}=F(1,1)+4 \pi \int\left(\rho_{1,1}^{\mathrm{F}}\right)^{2}$, and $[t]_{+}=t$ for $t>0$ and 0 otherwise. Moreover,

$$
\lim _{N a \rightarrow \infty} \rho_{1, N a}^{\mathrm{GP}}(x)=\rho_{1,1}^{\mathrm{F}}(x)
$$

strongly in $L^{2}\left(\mathbb{R}^{3}\right)$.

Proof. Since $\mathcal{E}^{\mathrm{GP}}[\sqrt{\rho}] \geq \mathcal{F}[\rho]$ it is clear that $E^{\mathrm{GP}}(1, N a) \geq F(1, N a)$. For the converse we write $\rho$ in the form $\rho(x)=(N a)^{-3 /(s+3)} \widetilde{\rho}\left((N a)^{-1 /(s+3)} x\right)$ and obtain

$$
\begin{aligned}
\mathcal{E}^{\mathrm{GP}}[\sqrt{\rho}] & =(N a)^{s /(s+3)} \int\left((N a)^{-(s+2) /(s+3)}|\nabla \sqrt{\widetilde{\rho}}|^{2}+V \widetilde{\rho}+4 \pi \widetilde{\rho}^{2}\right) d^{3} x \\
\mathcal{F}[\rho] & =(N a)^{s /(s+3)} \int\left(V \widetilde{\rho}+4 \pi \widetilde{\rho}^{2}\right) d^{3} x .
\end{aligned}
$$

In particular, $F(1, N a)=(N a)^{s /(s+3)} F(1,1)$, and with $\widetilde{\rho}=\rho_{1,1}^{\mathrm{F}}$ we obtain

$$
E^{\mathrm{GP}}(1, N a) \leq F(1, N a)+(N a)^{-2 /(s+3)} \int\left|\nabla \sqrt{\rho_{1,1}^{\mathrm{F}}}\right|^{2} .
$$

(Regularizing $V$, if necessary, we may assume that $\int\left|\nabla \sqrt{\rho_{1,1}^{\mathrm{F}}}\right|^{2}<\infty$.) In the limit $N a \rightarrow \infty$ the gradient term vanishes, and thus the limit of the energies is proved. Now

$$
\begin{aligned}
& \lim _{N a \rightarrow \infty} \frac{\mathcal{E}^{\mathrm{GP}}\left[\sqrt{\rho_{1, N a}^{\mathrm{GP}}}\right]}{(N a)^{s /(s+3)}}= \\
& \lim _{N a \rightarrow \infty}\left(\frac{\mathcal{F}\left[\rho_{1, N a}^{\mathrm{GP}}\right]}{(N a)^{s / s+3)}}+(N a)^{-\frac{s+2}{s+3}} \int\left|\nabla \sqrt{\rho_{1, N a}^{\mathrm{GP}}}\right|^{2}\right)=F(1,1) .
\end{aligned}
$$


Since $F(1,1)$ is the minimum of $\mathcal{F} /(N a)^{s /(s+3)}$, the second term vanishes for $N a \rightarrow \infty$, and it follows that $\rho_{1, N a}^{\mathrm{GP}}$ is a minimizing sequence for $\int\left(V \rho+4 \pi \rho^{2}\right)$. Since both terms in the functional are nonnegative, they must converge individually, in particular $\left\|\rho_{1, N a}^{\mathrm{GP}}\right\|_{2}$ converges to $\left\|\rho_{1,1}^{\mathrm{F}}\right\|_{2}$. On the other hand $\rho_{1, N a}^{\mathrm{GP}}$ converges weakly to $\rho_{1,1}^{\mathrm{F}}$ by uniqueness of the minimizer. Together with the convergence of the norms this implies strong convergence.

The solution of the variational equation for $\rho_{1,1}^{\mathrm{F}}$ is simply $\rho_{1,1}^{\mathrm{F}}=(8 \pi)^{-1}[\widetilde{\mu}-$ $V]_{+}$with $\widetilde{\mu}$ given by $\widetilde{\mu}=F(1,1)+4 \pi \int\left(\rho_{1,1}^{\mathrm{F}}\right)^{2}$.

Lemma 2.1 (Virial theorem). When $V$ is homogeneous of order $s$, as in (2.0), the minimizer of the GP functional satisfies

$$
\frac{2}{3} \int\left|\nabla \Phi^{\mathrm{GP}}(x)\right|^{2} d^{3} x-\frac{s}{3} \int \Phi^{\mathrm{GP}}(x)^{2} V(x) d^{3} x+4 \pi a \int \Phi^{\mathrm{GP}}(x)^{4} d^{3} x=0 .
$$

Proof. Define $\Phi_{k}$ by

$$
\Phi_{k}(x)=k^{1 / 2} \Phi^{\mathrm{GP}}\left(k^{1 / 3} x\right) .
$$

Because $\Phi^{\mathrm{GP}}$ is the minimizer of $\mathcal{E}^{\mathrm{GP}}[\Phi]$, it must be true that

$$
\left.\frac{\partial}{\partial k} \mathcal{E}^{\mathrm{GP}}\left[\Phi_{k}\right]\right|_{k=1}=0 .
$$

This leads to the virial theorem (2.11).

In the proof of the lower bound we shall also consider the GP energy functional in a finite box. For $R>0$ we denote by $\Lambda_{R}$ a cube centered at the origin, with side length $2 R$. The energy functional $\mathcal{E}_{R}^{\mathrm{GP}}$ in the box is simply (1.4) with the integration reduced to $\Lambda_{R}$, the corresponding minimizer, denoted by $\Phi_{R}^{\mathrm{GP}}$, satisfies Neumann conditions at the boundary of $\Lambda_{R}$, and is strictly positive. Analogously to (1.7), (1.9) and (2.5) we define $\rho_{R}^{\mathrm{GP}}, \bar{\rho}_{R}$ and $\mu_{R}$. The corresponding energy will be denoted by $E_{R}^{\mathrm{GP}}(N, a)$. Then we have the following

Lemma 2.2 (GP energy in a box).

$$
\lim _{R \rightarrow \infty} E_{R}^{\mathrm{GP}}(N, a)=E^{\mathrm{GP}}(N, a)
$$


Proof. Using $N^{1 / 2} \Phi^{\mathrm{GP}} \chi_{R} /\left\|\Phi^{\mathrm{GP}} \chi_{R}\right\|_{2}$ as a test function for $\mathcal{E}_{R}^{\mathrm{GP}}$, where $\chi_{R}$ denotes the characteristic function of $\Lambda_{R}$, we immediately get

$$
\lim _{R \rightarrow \infty} E_{R}^{\mathrm{GP}}(N, a) \leq E^{\mathrm{GP}}(N, a) .
$$

Let $\Theta_{R}$ be a $C^{\infty}$ function on $\mathbb{R}^{3}$, with $\Theta_{R}=0$ outside $\Lambda_{R}$, and $\Theta_{R}=1$ inside $\Lambda_{R-1}$. We use $N^{1 / 2} \Phi_{R}^{\mathrm{GP}} \Theta_{R} /\left\|\Phi_{R}^{\mathrm{GP}} \Theta_{R}\right\|_{2}$ as a test function for $\mathcal{E}^{\mathrm{GP}}$. Since $\nabla \Theta_{R}$ is bounded and

$$
\lim _{R \rightarrow \infty} \int_{\Lambda_{R} \backslash \Lambda_{R-1}}\left|\Phi_{R}^{\mathrm{GP}}\right|^{2}=0
$$

(because $V$ tends to infinity and $\mathcal{E}_{R}^{\mathrm{GP}}\left[\Phi_{R}^{\mathrm{GP}}\right]$ is bounded by (2.13)), we have

$$
E^{\mathrm{GP}}(N, a) \leq \liminf _{R \rightarrow \infty} E_{R}^{\mathrm{GP}}(N, a)
$$

This completes the proof of (2.12).

\section{$3 \quad$ Upper Bounds}

\subsection{Upper bound for the QM energy}

It will now be shown that for all $N$ and small values of $a \bar{\rho}^{1 / 3}$ (with $\bar{\rho}=$ $\int \rho^{\mathrm{GP}}(x)^{2} d^{3} x / N$, cf. (1.9))

$$
E^{\mathrm{QM}}(N, a) \leq E^{\mathrm{GP}}(N, a)\left(1+O\left(a \bar{\rho}^{\frac{1}{3}}\right)\right) .
$$

This upper bound, which holds for all positive, spherically symmetric $v$ with finite scattering length, is derived by means of the variational principle. We generalize a method of Dyson [6], who proved an upper bound for the homogeneous Bose gas with hard-sphere interaction. Consider as a trial function

$$
\Psi=F\left(x_{1}, \ldots, x_{N}\right) G\left(x_{1}, \ldots, x_{N}\right)
$$

with

$$
F\left(x_{1}, \ldots, x_{N}\right)=\prod_{i=1}^{N} F_{i}\left(x_{1}, \ldots, x_{i}\right), \quad G\left(x_{1}, \ldots, x_{N}\right)=\prod_{i=1}^{N} g\left(x_{i}\right)
$$


where

$$
F_{i}\left(x_{1}, \ldots, x_{i}\right)=f\left(t_{i}\right), \quad t_{i}=\min \left(\left|x_{i}-x_{j}\right|, j=1, \ldots, i-1\right),
$$

with a function $f$ satisfying

$$
0 \leq f \leq 1, \quad f^{\prime} \geq 0
$$

and

$$
g(x)=\Phi^{\mathrm{GP}}(x) /\left\|\Phi^{\mathrm{GP}}\right\|_{\infty} .
$$

The function $f$ will be specified later. This trial function is not symmetric in the particle coordinates, but the expectation value $\left\langle\Psi \mid H^{(N)} \Psi\right\rangle /\langle\Psi \mid \Psi\rangle$ is still an upper bound to the bosonic ground state energy because the Hamiltonian is symmetric and its ground state wave function is positive. Hence the bosonic ground state energy is equal to the absolute ground state energy ([6], [10]).

The physical meaning of the trial function can be understood as follows: The $G$ part describes independent particles, each with the GP wave function. The $F$ part means that the particles are inserted into the system one at a time, taking into account the particles previously inserted, but without adjusting their wave function (cf. [6]). Although a wave function of this form cannot describe all correlations present in the true ground state, it captures the leading term in the energy for dilute systems.

For the computation of the kinetic energy we use

$$
\int_{\mathbb{R}^{3 N}} \Psi \nabla_{k}^{2} \Psi=\int_{\mathbb{R}^{3 N}}\left(G \nabla_{k}^{2} G\right) F^{2}-\int_{\mathbb{R}^{3 N}} G^{2}\left|\nabla_{k} F\right|^{2},
$$

where $\nabla_{k}$ denotes the gradient with respect to $x_{k}, k=1, \ldots, N$. We write

$$
\epsilon_{i k}= \begin{cases}1 & \text { for } i=k \\ -1 & \text { for } t_{i}=\left|x_{i}-x_{k}\right| \\ 0 & \text { otherwise }\end{cases}
$$

Let $n_{i}$ be the unit vector in the direction of $x_{i}-x_{j(i)}$, when $x_{j(i)}$ is the nearest to $x_{i}$ of the points $\left(x_{1}, \ldots, x_{i-1}\right)$. (Note that $j(i)$ really depends on all the 
points $x_{1}, \ldots, x_{i}$ and not just on the index $i$. Except for a set of zero measure, $j(i)$ is unique.) Then

$$
G \nabla_{k} F=\sum_{i} \Psi F_{i}^{-1} \epsilon_{i k} n_{i} f^{\prime}\left(t_{i}\right)
$$

and after summation over $k$

$$
\begin{aligned}
& \sum_{k} G^{2}\left|\nabla_{k} F\right|^{2}=|\Psi|^{2} \sum_{i, j, k} \epsilon_{i k} \epsilon_{j k}\left(n_{i} \cdot n_{j}\right) F_{i}^{-1} F_{j}^{-1} f^{\prime}\left(t_{i}\right) f^{\prime}\left(t_{j}\right) \\
& \leq 2|\Psi|^{2} \sum_{i} F_{i}^{-2} f^{\prime}\left(t_{i}\right)^{2}+2|\Psi|^{2} \sum_{k \leq i<j}\left|\epsilon_{i k} \epsilon_{j k}\right| F_{i}^{-1} F_{j}^{-1} f^{\prime}\left(t_{i}\right) f^{\prime}\left(t_{j}\right) .
\end{aligned}
$$

The expectation value can thus be bounded as follows:

$$
\begin{aligned}
\frac{\left\langle\Psi \mid H^{(N)} \Psi\right\rangle}{\langle\Psi \mid \Psi\rangle} & \leq 2 \sum_{i=1}^{N} \frac{\int|\Psi|^{2} F_{i}^{-2} f^{\prime}\left(t_{i}\right)^{2}}{\int|\Psi|^{2}}+\sum_{j<i} \frac{\int|\Psi|^{2} v\left(\left|x_{i}-x_{j}\right|\right)}{\int|\Psi|^{2}} \\
& +2 \sum_{k \leq i<j} \frac{\int|\Psi|^{2}\left|\epsilon_{i k} \epsilon_{j k}\right| F_{i}^{-1} F_{j}^{-1} f^{\prime}\left(t_{i}\right) f^{\prime}\left(t_{j}\right)}{\int|\Psi|^{2}} \\
& +\sum_{i=1}^{N} \frac{\int|\Psi|^{2}\left(-g\left(x_{i}\right)^{-1} \nabla_{i}^{2} g\left(x_{i}\right)+V\left(x_{i}\right)\right)}{\int|\Psi|^{2}} .
\end{aligned}
$$

For $i<p$, let $F_{p, i}$ be the value that $F_{p}$ would take if the point $x_{i}$ were omitted, i.e.,

$$
F_{p, i}=f\left(\left|x_{p}-x_{k(p)}\right|\right),
$$

where $x_{k(p)}$ is the nearest to $x_{p}$ of the points $\left(x_{1}, \ldots, x_{i-1}, x_{i+1}, \ldots, x_{p-1}\right)$. The reason for introducing these functions is that one wants to decouple the integration over $x_{i}$ from the integrations over the other variables. (Note that $F_{p, i}$ is independent of $x_{i}$.) Analogously, one defines $F_{p, i j}$ by omitting $x_{i}$ and $x_{j}$. This decouples simultaneously $x_{i}$ and $x_{j}$ from the other variables. The functions $F_{i}$ occur both in the numerator and the denominator so one needs estimates from below and above. Since

$$
F_{p}=\min \left\{F_{p, i j}, f\left(\left|x_{p}-x_{j}\right|\right), f\left(\left|x_{p}-x_{i}\right|\right)\right\},
$$

one has (recall that $f \leq 1$ )

$$
F_{p, i j}^{2} f\left(\left|x_{p}-x_{i}\right|\right)^{2} f\left(\left|x_{p}-x_{j}\right|\right)^{2} \leq F_{p}^{2} \leq F_{p, i j}^{2}
$$


Hence, with $j<i$,

$$
F_{j+1}^{2} \ldots F_{i-1}^{2} F_{i+1}^{2} \ldots F_{N}^{2} \leq F_{j+1, j}^{2} \ldots F_{i-1, j}^{2} F_{i+1, i j}^{2} \ldots F_{N, i j}^{2}
$$

and

$$
\begin{aligned}
& F_{j}^{2} \ldots F_{N}^{2} \geq F_{j+1, j}^{2} \ldots F_{i-1, j}^{2} F_{i+1, i j}^{2} \ldots F_{N, i j}^{2} \\
& \cdot\left(1-\sum_{k=1, k \neq i, j}^{N}\left(1-f\left(\left|x_{j}-x_{k}\right|\right)^{2}\right)\right) \cdot\left(1-\sum_{k=1, k \neq i}^{N}\left(1-f\left(\left|x_{i}-x_{k}\right|\right)^{2}\right)\right) .
\end{aligned}
$$

We now consider the first two terms in (3.11). In the numerator of the first term for each fixed $i$ we use the estimate

$$
f^{\prime}\left(t_{i}\right)^{2} \leq \sum_{j=1}^{i-1} f^{\prime}\left(\left|x_{i}-x_{j}\right|\right)^{2}
$$

and in the second term we use $F_{i} \leq f\left(\left|x_{i}-x_{j}\right|\right)$. For fixed $i$ and $j$ one eliminates $x_{i}$ and $x_{j}$ from the rest of the integrand by using (3.15) and $F_{j} \leq 1$ in the numerator and (3.16) in the denominator to do the $x_{i}$ and $x_{j}$ integrations. With the transformation $\eta=x_{i}-x_{j}, \chi=\left(x_{i}+x_{j}\right) / 2$ one gets

$$
\begin{aligned}
& \int\left(2 f^{\prime}\left(\left|x_{i}-x_{j}\right|\right)^{2}+v\left(\left|x_{i}-x_{j}\right|\right) f\left(\left|x_{i}-x_{j}\right|\right)^{2}\right) g\left(x_{i}\right)^{2} g\left(x_{j}\right)^{2} d^{3} x_{i} d^{3} x_{j}= \\
& \int\left(2 f^{\prime}(|\eta|)^{2}+v(|\eta|) f(|\eta|)^{2}\right) g\left(\chi+\frac{1}{2} \eta\right)^{2} g\left(\chi-\frac{1}{2} \eta\right)^{2} d^{3} \eta d^{3} \chi
\end{aligned}
$$

By the Cauchy-Schwarz inequality

$$
\int g\left(\chi+\frac{1}{2} \eta\right)^{2} g\left(\chi-\frac{1}{2} \eta\right)^{2} d^{3} \chi \leq \int g(\chi)^{4} d^{3} \chi
$$

and one obtains

$$
\begin{aligned}
& \int\left(2 f^{\prime}\left(\left|x_{i}-x_{j}\right|\right)^{2}+v\left(\left|x_{i}-x_{j}\right|\right) f\left(\left|x_{i}-x_{j}\right|\right)^{2}\right) g\left(x_{i}\right)^{2} g\left(x_{j}\right)^{2} d^{3} x_{i} d^{3} x_{j} \\
& \leq \int g(\chi)^{4} d^{3} \chi \int\left(2 f^{\prime}(|\eta|)^{2}+v(|\eta|) f(|\eta|)^{2}\right) d^{3} \eta \equiv 2 \int g(\chi)^{4} d^{3} \chi J
\end{aligned}
$$


In the denominator one gets, using that $0 \leq g \leq 1$,

$$
\begin{aligned}
& \int\left(1-\sum_{p=1, p \neq i}^{N}\left(1-f\left(\left|x_{p}-x_{i}\right|\right)^{2}\right)\right) g\left(x_{i}\right)^{2} d^{3} x_{i} \\
& \geq \int g(x)^{2} d^{3} x-N \int\left(1-f\left(\left|x_{p}-x_{i}\right|\right)^{2}\right) \equiv \int g(x)^{2} d^{3} x-N I .
\end{aligned}
$$

The same factor comes from the $x_{j}$-integration, the remaining factors are identical in numerator and denominator, and so finally the first and second term are bounded by

$$
\sum_{i=1}^{N}(i-1) \frac{2 \int g(x)^{4} d^{3} x J}{\left(\int g(x)^{2} d^{3} x-N I\right)^{2}} \leq N^{2} \frac{\int g(x)^{4} d^{3} x J}{\left(\int g(x)^{2} d^{3} x-N I\right)^{2}}
$$

A similar argument is now applied to the third term of (3.11). Note that the contributions from $k=i$ and $k<i$ are the same. Therefore

$$
\begin{aligned}
& \sum_{k=1}^{i} \int\left|\epsilon_{i k}\right| f\left(t_{i}\right) f^{\prime}\left(t_{i}\right)\left|\epsilon_{j k}\right| f\left(t_{j}\right) f^{\prime}\left(t_{j}\right) g\left(x_{i}\right)^{2} g\left(x_{j}\right)^{2} d^{3} x_{i} d^{3} x_{j} \leq \\
& 2 \sum_{k=i}^{i-1} \int f\left(\left|x_{i}-x_{k}\right|\right) f^{\prime}\left(\left|x_{i}-x_{k}\right|\right) f\left(\left|x_{j}-x_{k}\right|\right) f^{\prime}\left(\left|x_{j}-x_{k}\right|\right) g\left(x_{i}\right)^{2} g\left(x_{j}\right)^{2} d^{3} x_{i} d^{3} x_{j} .
\end{aligned}
$$

With $g \leq 1$ one gets

$$
\begin{aligned}
& 2 \sum_{k=1}^{i-1} \int f\left(\left|x_{i}\right|\right) f^{\prime}\left(\left|x_{i}\right|\right) f\left(\left|x_{j}\right|\right) f^{\prime}\left(\left|x_{j}\right|\right) d^{3} x_{i} d^{3} x_{j} \\
& =2(i-1)\left(\int f(|x|) f^{\prime}(|x|) d^{3} x\right)^{2} \equiv 2(i-1) K^{2}
\end{aligned}
$$

The summation over $i$ and $j$ gives

$$
\sum_{j=1}^{N} \sum_{i=1}^{j-1}(i-1)=\frac{1}{6} N(N-1)(N-2) .
$$

In the denominator one gets the same factors as above, and so the third term is bounded by

$$
\frac{2}{3} N^{3} \frac{K^{2}}{\left(\int g(x)^{2} d^{3} x-N I\right)^{2}}
$$


Next consider the last term of (3.11). Define $\widetilde{e}$ by

$$
\int\left(-g(x) \nabla^{2} g(x)+V(x) g(x)^{2}\right) d^{3} x \equiv \widetilde{e} \int g(x)^{2} d^{3} x .
$$

After eliminating $x_{i}$ from the integrands in the numerator and the denominator and using $F_{i} \leq 1$ one sees that the term is bounded above by

$$
N \frac{\widetilde{e} \int g(x)^{2} d^{3} x}{\int g(x)^{2} d^{3} x-N I}
$$

Putting all terms together we obtain as an upper bound for the ground state energy

$\frac{E^{\mathrm{QM}}}{N} \leq \frac{\widetilde{e} \int g(x)^{2} d^{3} x}{\int g(x)^{2} d^{3} x-N I}+N \frac{\int g(x)^{4} d^{3} x J}{\left(\int g(x)^{2} d^{3} x-N I\right)^{2}}+\frac{2}{3} N^{2} \frac{K^{2}}{\left(\int g(x)^{2} d^{3} x-N I\right)^{2}}$

with $I, J, K$ and $\widetilde{e}$ defined by (3.21), (3.20), (3.24) and (3.27). It remains to choose $f$. We take for $b>a$ (we shall soon fix $b$ )

$$
f(r)= \begin{cases}(1+\epsilon) u(r) / r & \text { for } r \leq b \\ 1 & \text { for } r>b\end{cases}
$$

where $u(r)$ is the solution of the scattering equation

$$
-u^{\prime \prime}(r)+\frac{1}{2} v(r) u(r)=0 \quad \text { with } u(0)=0, \lim _{r \rightarrow \infty} u^{\prime}(r)=1
$$

and $\epsilon$ is determined by requiring $f$ to be continuous. Convexity of $u$ gives

$$
r \geq u(r) \geq\left\{\begin{array}{ll}
0 & \text { for } r \leq a \\
r-a & \text { for } r>a
\end{array}, \quad 1 \geq u^{\prime}(r) \geq\left\{\begin{array}{ll}
0 & \text { for } r \leq a \\
1-\frac{a}{r} & \text { for } r>a
\end{array} .\right.\right.
$$

These estimates imply

$$
\begin{aligned}
& J \leq(1+\epsilon)^{2} 4 \pi a \\
& I \leq 4 \pi\left(\frac{a^{3}}{3}+a b(b-a)\right) \\
& K \leq 4 \pi(1+\epsilon) a\left(b-\frac{a}{2}\right) \\
& 0 \leq \epsilon \leq \frac{a}{b-a}
\end{aligned}
$$


For (3.33) we used partial integration. By definition (1.9)

$$
\bar{\rho}=\frac{1}{N} \int\left(\rho^{\mathrm{GP}}\right)^{2}=N \frac{\int g^{4}}{\left(\int g^{2}\right)^{2}}
$$

and we choose $b$ such that

$$
\frac{4 \pi}{3} \bar{\rho} b^{3}=\frac{\int g^{4}}{\int g^{2}}=\frac{\bar{\rho}}{\left\|\rho^{\mathrm{GP}}\right\|_{\infty}} \equiv c .
$$

With this choice the factor in the denominators in (3.29) is bounded by

$$
\int g^{2}-N I \geq \int g^{2}\left(1-\frac{a}{b}\right)^{3} .
$$

Note that $c \leq 1$, and $a<b$ holds provided

$$
\frac{a^{3}}{b^{3}}=\frac{4 \pi}{3} a^{3}\left\|\rho^{\mathrm{GP}}\right\|_{\infty}<1
$$

Collecting the estimates (3.33)-(3.36), we finally obtain

Theorem 3.1 (Upper bound for the QM energy).

$E^{\mathrm{QM}} \leq \frac{\int\left(\left|\nabla \Phi^{\mathrm{GP}}\right|^{2}+V\left(\Phi^{\mathrm{GP}}\right)^{2}\right)}{\left(1-\frac{a}{b}\right)^{3}}+4 \pi a \int\left(\Phi^{\mathrm{GP}}\right)^{4} \frac{1+\frac{2}{c}\left(\frac{a}{b}\right)-\frac{2}{c}\left(\frac{a}{b}\right)^{2}+\frac{1}{2 c}\left(\frac{a}{b}\right)^{3}}{\left(1-\frac{a}{b}\right)^{8}}$

with $b$ and $c$ defined by (3.38).

Remark 3.1 (Negative potentials with hard core). (3.1) can be extended to include partially negative potentials of the form

$$
v(r)= \begin{cases}\infty & \text { for } 0 \leq r \leq d \\ -|w(r)| & \text { for } d<r \leq R_{0} \\ 0 & \text { for } r>R_{0}\end{cases}
$$

as long as $f^{\prime}(r)^{2}+\frac{1}{2} v(r) f(r)^{2} \geq 0$ for all $r$. With $f$ from (3.30), this is the case for sufficient shallow potentials. The potential energy is then negative, and the estimates used for (3.18) are no longer valid. But

$$
\sum_{j<i} v\left(\left|x_{i}-x_{j}\right|\right) F_{i}^{2} \leq \sum_{i} v\left(t_{i}\right) F_{i}^{2}
$$


and because of $2 f^{\prime}(r)^{2}+v(r) f(r)^{2} \geq 0$ we get

$$
2 f^{\prime}\left(t_{i}\right)^{2}+v\left(t_{i}\right) f\left(t_{i}\right)^{2} \leq \sum_{j=1}^{i-1} 2 f^{\prime}\left(\left|x_{i}-x_{j}\right|\right)^{2}+v\left(\left|x_{i}-x_{j}\right|\right) f\left(\left|x_{i}-x_{j}\right|\right)^{2} .
$$

So we have the same as in (3.18). Note that for potentials as in (3.42) $f$ satisfies (3.5), as long as $a>0$.

Remark 3.2 (Homogeneous gas). For the special case of a homogeneous Bose gas (i.e. $V=0$ ) in a box of volume $\mathcal{V}$, the GP density is simply

$$
\rho^{\mathrm{GP}}(x)=N / \mathcal{V}=\bar{\rho},
$$

for all $x$ in the box, and the GP energy is given by

$$
E^{\mathrm{GP}}(N, a)=4 \pi a \frac{N^{2}}{\mathcal{V}}
$$

Our method also applies here, if we impose periodic boundary conditions on the box. Therefore our upper bound is a generalization of a result by Dyson [6], who proved an analogous bound for the special case of a homogeneous Bose gas with hard-sphere interaction.

\subsection{Upper bound for the chemical potential}

By the same method as in the previous subsection one can derive a bound on the increase of the energy when one particle is added to the system. This bound will be needed for the derivation of the lower bound to the energy.

Theorem 3.2 (Upper bound for the chemical potential). Let $E^{*}(N, a)$ denote the infimum of the functional

$$
\mathcal{E}^{*}[\Phi]=\int\left(|\nabla \Phi(x)|^{2}+V(x)|\Phi(x)|^{2}+8 \pi a\|\Phi\|_{\infty}^{2}|\Phi(x)|^{2}\right) d^{3} x
$$

with $\int|\Phi|^{2}=N$. Let $\Phi^{*}$ be the positive minimizer of $\mathcal{E}^{*}$ (its existence is guaranteed by the same arguments as for the GP functional itself), and $\bar{\rho}^{*}=$ $\int \Phi^{* 4} / N$. Then

$$
E^{\mathrm{QM}}(N+1, a) \leq E^{\mathrm{QM}}(N, a)+E^{*}(1, N a)\left(1+O\left(a \bar{\rho}^{* 1 / 3}\right)\right) .
$$


Proof. Let $\Psi_{0}^{(N)}$ be the ground state wave function of $H^{(N)}$. As test wave function for $H^{(N+1)}$ we take

$$
\Psi\left(x_{1}, \ldots, x_{N+1}\right)=\Psi_{0}^{(N)}\left(x_{1}, \ldots, x_{N}\right) \Phi^{*}\left(x_{N+1}\right) f\left(t_{N+1}\right),
$$

where $f$ and $t_{N+1}$ are defined as in (3.30) and (3.4), i.e., $r f(r)$ is essentially the zero energy scattering solution and $t_{N+1}$ is the distance of $x_{N+1}$ from its nearest neighbor. We have

$$
\begin{aligned}
& \left\langle\Psi \mid H^{(N+1)} \Psi\right\rangle= \\
& \int f^{2} \Phi^{* 2}\left(\sum_{i=1}^{N}\left(-\overline{\Psi_{0}^{(N)}} \nabla_{i}^{2} \Psi_{0}^{(N)}+V\left(x_{i}\right)\left|\Psi_{0}^{(N)}\right|^{2}\right)+\sum_{i<j}^{N} v\left(\left|x_{i}-x_{j}\right|\right)\left|\Psi_{0}^{(N)}\right|^{2}\right) \\
& +\int\left|\Psi_{0}^{(N)}\right|^{2} \Phi^{* 2}\left(\sum_{i=1}^{N}\left|\nabla_{i} f\right|^{2}\right)+\int\left|\Psi_{0}^{(N)}\right|^{2} f^{2}\left(-\Phi^{*} \nabla_{N+1}^{2} \Phi^{*}+V\left(x_{N+1}\right) \Phi^{* 2}\right) \\
& +\int\left|\Psi_{0}^{(N)}\right|^{2} \Phi^{* 2}\left(\left|\nabla_{N+1} f\right|^{2}+\sum_{i=1}^{N} v\left(\left|x_{N+1}-x_{i}\right|\right) f^{2}\right) .
\end{aligned}
$$

For $f$ one uses the estimates

$$
f \leq 1, \quad f\left(t_{N+1}\right)^{2} \geq 1-\sum_{i=1}^{N}\left(1-f\left(\left|x_{N+1}-x_{i}\right|\right)^{2}\right)
$$

and for the derivatives one has

$$
\left|\nabla_{N+1} f\right|^{2}=f^{\prime}\left(t_{N+1}\right)^{2}=\sum_{i=1}^{N}\left|\nabla_{i} f\right|^{2}
$$

and

$$
f^{\prime}\left(t_{N+1}\right)^{2} \leq \sum_{i=1}^{N} f^{\prime}\left(\left|x_{N+1}-x_{i}\right|\right)^{2}
$$


After division by the norm of $\Psi$ (3.50) becomes

$$
\begin{aligned}
& E^{\mathrm{QM}}(N+1, a) \leq E^{\mathrm{QM}}(N, a)+\frac{\int\left|\Psi_{0}^{(N)}\right|^{2}\left(-\Phi^{*} \nabla_{N+1}^{2} \Phi^{*}+V \Phi^{* 2}\right)}{\int\left|\Psi_{0}^{(N)}\right|^{2}\left(N-N \Phi^{* 2} \int\left(1-f^{2}\right)\right)} \\
& +\frac{\int\left|\Psi_{0}^{(N)}\right|^{2}\left\|\Phi^{*}\right\|_{\infty}^{2} \sum_{i=1}^{N}\left(2 f^{\prime}\left(\left|x_{N+1}-x_{i}\right|\right)^{2}+v\left(\left|x_{N+1}-x_{i}\right|\right) f\left(\left|x_{N+1}-x_{i}\right|\right)^{2}\right)}{\int\left|\Psi_{0}^{(N)}\right|^{2}\left(N-N\left\|\Phi^{*}\right\|_{\infty}^{2} \int\left(1-f^{2}\right)\right)} .
\end{aligned}
$$

$\Psi_{0}^{(N)}$ does not depend on $x_{N+1}$. One integrates first over $x_{N+1}$ and then over the remaining variables. In analogy with the estimates (3.33) and (3.39) one gets

$$
\int\left(2 f^{\prime}(|x|)^{2}+v(|x|) f(|x|)^{2}\right) d^{3} x \leq 8 \pi a\left(1+O\left(a \bar{\rho}^{* 1 / 3}\right)\right)
$$

and

$$
\left\|\Phi^{*}\right\|_{\infty}^{2} \int\left(1-f(|x|)^{2}\right) d^{3} x \leq O\left(a \bar{\rho}^{* 1 / 3}\right)
$$

This implies

$$
E^{\mathrm{QM}}(N+1, a) \leq E^{\mathrm{QM}}(N, a)+\frac{E^{*}(N, a)}{N}\left(1+O\left(a \bar{\rho}^{* 1 / 3}\right)\right) .
$$

By scaling, $E^{*}(N, a)=N E^{*}(1, N a)$ and (3.48) follows.

Remark 3.3 (Box with Neumann conditions). Eq. (3.48) also holds for a box with Neumann boundary conditions. To see this we note that Neumann conditions give the lowest energy for the quadratic form $\left\langle\Psi \mid H^{(N+1)} \Psi\right\rangle$, therefore it is possible to use (3.49) as a test function, even if $f$ does not fulfill Neumann conditions. If $\Psi_{0}^{(N)}$ and $\Phi^{*}$ do, the calculation above is still valid, since for

$$
\int|\nabla(g f)|^{2}=-\int f^{2} g \nabla^{2} g+\int g^{2}|\nabla f|^{2}
$$

only boundary conditions for $g$ are needed.

Note also that in the homogeneous case, i.e. $V=0$ in the box, $E^{*}(N, a)=$ $2 E^{\mathrm{GP}}(N, a)$. 


\section{Lower Bounds}

\subsection{The homogeneous case}

In [5] the following lower bound was established for the ground state energy, $E^{\text {hom }}$, of a Bose gas in a box of side length $L$ with Neumann boundary conditions and $v$ of finite range:

$$
E^{\mathrm{hom}}(N, L) \geq 4 \pi a \frac{N^{2}}{L^{3}}\left(1-C Y^{1 / 17}\right)
$$

with $Y=a^{3} N / L^{3}$ and $C$ a constant. The estimate holds for all $Y$ small enough and $L / a \gg Y^{-6 / 17}$ (note that this implies $N \gg Y^{-1 / 17}$ ). In the thermodynamic limit the constant $C$ can be taken to be $C=8.9$, but this value is only of academic interest, because the error term $-C Y^{1 / 17}$ is not believed to reflect the true state of affairs. Presumably, it does not even have the correct sign.

The restriction of a finite range can be relaxed. In fact, (4.1) holds (with a different constant $C$ ) for all positive, spherically symmetric $v$ with

$$
v(r) \leq \text { const. } r^{-\left(3+\frac{1}{5}+\epsilon\right)} \quad \text { for } r \text { large enough, } \epsilon>0 .
$$

More generally, if

$$
v(r) \leq \text { const. } r^{-(3+\epsilon)} \quad \text { for } r \text { large enough, } \epsilon>0
$$

then (4.1) holds at least with the exponent $1 / 17$ replaced by an exponent $O(\epsilon)$. We prove these assertions in Appendix B.

In the next section we shall stick to the estimate (4.1) for simplicity, but in the limit $N \rightarrow \infty$ the explicit form of the error term is not significant so a decrease of the potential as in (4.3) is sufficient for the limit Theorems 1.1 and 1.2 .

\subsection{The lower bound in the inhomogeneous case}

Our generalization of (4.1) to the inhomogeneous case is as follows:

Theorem 4.1 (Lower bound for the QM energy). Let $v$ be positive, spherically symmetric and decrease at infinity like (4.2). Its scattering length is $a=a_{1} / N$ with $a_{1}$ fixed, as explained in the Introduction. Then as $N \rightarrow \infty$

$$
E^{\mathrm{QM}}(N, a) \geq E_{R}^{\mathrm{GP}}(N, a)\left(1-\text { const. } N^{-1 / 10}\right)
$$


for all $R$ large enough, where $E_{R}^{\mathrm{GP}}$ is the GP energy in a cube with side length $2 R$, center at the origin, and Neumann boundary conditions; the constant in (4.4) depends only on $a_{1}$ and $R$.

Proof. As in [5] the lower bound will be obtained by dividing space into cubic boxes with Neumann conditions at the boundary, which only lowers the energy. Moreover, interactions among particles in different boxes are dropped. Since $v \geq 0$, this, too, lowers the energy. For the lower bound one has to estimate the energy for a definite particle number in each box and then to optimize over all distributions of the $N$ particles among the boxes.

Step 1 (Finite box): The first step is to show that all the particles can be assumed to be in some large, but finite box. Since

$$
K(R)=\inf _{|x|>R} V(x)
$$

tends monotonically to $\infty$ with $R$, one knows that the energy of a particle outside a cube $\Lambda_{R}$ of side length $2 R$ and center at the origin is at least $K(R)$. Hence

$$
E^{\mathrm{QM}}(N, a) \geq \inf _{0 \leq n \leq N}\left\{E_{R}^{\mathrm{QM}}(N-n, a)+n K(R)\right\},
$$

where $E_{R}^{\mathrm{QM}}(N-n, a)$ denotes the energy of $N-n$ particles in $\Lambda_{R}$, with Neumann conditions at the boundary. We now apply Theorem 3.2 (which holds also in a cube with Neumann conditions). Applying the theorem $n$ times and noting that $E^{*}(1, N a)$ is monotone in $N$ we have

$$
E_{R}^{\mathrm{QM}}(N-n, a) \geq E_{R}^{\mathrm{QM}}(N, a)-n E^{*}(1, N a)\left(1+O\left(a \bar{\rho}^{* 1 / 3}\right)\right) .
$$

Hence there is a constant $K^{\prime}$ (that depends only on $N a$ ), such that $K(R)>$ $K^{\prime}$ implies that the infimum is obtained at $n=0$. This is fulfilled for all sufficiently large $R$, independently of $N$ if $N a$ is fixed. So we can restrict ourselves to estimating the energy in $\Lambda_{R}$ with Neumann boundary conditions.

Step 2 (Trading $V$ for $-\rho_{R}^{\mathrm{GP}}$ ): We shall now use the GP equation to eliminate $V$ from the problem, effectively replacing it by $-8 \pi a \rho_{R}^{\mathrm{GP}}$. We write the wave function in $\Lambda_{R}^{N}$ as

$$
\Psi\left(x_{1}, \ldots, x_{N}\right)=f\left(x_{1}, \ldots, x_{N}\right) \prod_{i=1}^{N} \Phi_{R}^{\mathrm{GP}}\left(x_{i}\right),
$$


where $\Phi_{R}^{\mathrm{GP}}$ denotes the the minimizer of the GP functional in $\Lambda_{R}$; since it is strictly positive, every wave function can be written in this form. Note also that $\Phi_{R}^{\mathrm{GP}}$ and $f$ obey Neumann conditions. We have

$$
\begin{aligned}
\langle\Psi \mid H \Psi\rangle & =\sum_{i=1}^{N} \int|\Psi|^{2} \Phi_{R}^{\mathrm{GP}}\left(x_{i}\right)^{-1}\left(-\nabla_{i}^{2}+V\left(x_{i}\right)\right) \Phi_{R}^{\mathrm{GP}}\left(x_{i}\right) d^{3 N} x \\
& +\sum_{i=1}^{N} \int \prod_{k=1}^{N} \Phi_{R}^{\mathrm{GP}}\left(x_{k}\right)^{2}\left|\nabla_{i} f\right|^{2} d^{3 N} x+\sum_{i<j}^{N} \int|\Psi|^{2} v\left(\left|x_{i}-x_{j}\right|\right) d^{3 N} x,
\end{aligned}
$$

where the integrals are over $\Lambda_{R}^{N}$. Using the GP equation (2.4) this becomes

$$
\begin{aligned}
& \langle\Psi \mid H \Psi\rangle= \\
& \sum_{i=1}^{N} \int \prod_{k=1}^{N} \rho_{R}^{\mathrm{GP}}\left(x_{k}\right)\left(\left|\nabla_{i} f\right|^{2}+\left(\mu_{R}-8 \pi a \rho_{R}^{\mathrm{GP}}\left(x_{i}\right)+\sum_{j=1}^{i-1} v\left(\left|x_{i}-x_{j}\right|\right)\right)|f|^{2}\right) .
\end{aligned}
$$

Inserting the value (2.5) for $\mu_{R}$ gives

$$
\begin{gathered}
\frac{\langle\Psi \mid H \Psi\rangle}{\langle\Psi \mid \Psi\rangle}-E_{R}^{\mathrm{GP}}=4 \pi a \bar{\rho}_{R} N+Q(f) \\
Q(f)=\sum_{i=1}^{N} \frac{\int_{\Lambda_{R}^{N}} \prod_{k=1}^{N} \rho_{R}^{\mathrm{GP}}\left(x_{k}\right)\left(\left|\nabla_{i} f\right|^{2}+\sum_{j=1}^{i-1} v\left(\left|x_{i}-x_{j}\right|\right)|f|^{2}-8 \pi a \rho_{R}^{\mathrm{GP}}\left(x_{i}\right)|f|^{2}\right)}{\int_{\Lambda_{R}^{N}} \prod_{k=1}^{N} \rho_{R}^{\mathrm{GP}}\left(x_{k}\right)|f|^{2}} .
\end{gathered}
$$

Step 3 (Division into boxes): $Q(f)$ is a normalized quadratic form on the weighted $L^{2}$-space $L^{2}\left(\Lambda_{R}^{N}, \prod_{k=1}^{N} \rho_{R}^{\mathrm{GP}}\left(x_{k}\right) d^{3} x_{k}\right)$, and can be minimized by dividing the cube $\Lambda_{R}$ into smaller cubes with side length L, labelled by $\alpha$, distributing the $N$ particles among the boxes and optimizing over all distributions. We therefore have

$$
\inf _{f} Q(f) \geq \inf _{\left\{n_{\alpha}\right\}} \sum_{\alpha} \inf _{f_{\alpha}} Q_{\alpha}\left(f_{\alpha}\right)
$$


where the infimum is taken over all distributions of the particles with $\sum n_{\alpha}=N$, and $Q_{\alpha}(f)$ is given by

$Q_{\alpha}(f)=\sum_{i=1}^{n_{\alpha}} \frac{\int \prod_{\alpha=1}^{n_{\alpha}} \rho_{R}^{\mathrm{GP}}\left(x_{k}\right)\left(\left|\nabla_{i} f\right|^{2}+\sum_{j=1}^{i-1} v\left(\left|x_{i}-x_{j}\right|\right)|f|^{2}-8 \pi a \rho_{R}^{\mathrm{GP}}\left(x_{i}\right)|f|^{2}\right)}{\int \prod_{\alpha=1}^{n_{\alpha}} \rho_{R}^{\mathrm{GP}}\left(x_{k}\right)|f|^{2}}$,

where the integrals are over $x_{k}$ in the box $\alpha, k=1, \ldots, n_{\alpha}$. Note that here $f=f\left(x_{1}, \ldots, x_{n_{\alpha}}\right)$, and (4.14) is the same as (4.12) with $N$ replaced by $n_{\alpha}$ and $\Lambda_{R}$ with the box $\alpha$.

We now want to use (4.1) and therefore we must approximate $\rho_{R}^{\text {GP }}$ by constants in each box. Let $\rho_{\alpha, \max }$ and $\rho_{\alpha, \min }$, respectively, denote the maximal and minimal values of $\rho_{R}^{\mathrm{GP}}$ in box $\alpha$. With

$$
\Phi^{(i)}\left(x_{1}, \ldots, x_{n_{\alpha}}\right)=f\left(x_{1}, \ldots, x_{n_{\alpha}}\right) \prod_{\substack{k=1 \\ k \neq i}}^{n_{\alpha}} \Phi_{R}^{\mathrm{GP}}\left(x_{k}\right),
$$

one has

$$
\begin{aligned}
& \frac{\int \prod_{k} \rho_{R}^{\mathrm{GP}}\left(x_{k}\right)\left(\left|\nabla_{i} f\right|^{2}+\sum_{j=1}^{i-1} v\left(\left|x_{i}-x_{j}\right|\right)|f|^{2}\right)}{\int \prod_{k} \rho_{R}^{\mathrm{GP}}\left(x_{k}\right)|f|^{2}} \\
& \geq \frac{\rho_{\alpha, \min }}{\rho_{\alpha, \max }} \frac{\int\left|\nabla_{i} \Phi^{(i)}\right|^{2}+\sum_{j=1}^{i-1} v\left(\left|x_{i}-x_{j}\right|\right)\left|\Phi^{(i)}\right|^{2}}{\int\left|\Phi^{(i)}\right|^{2}} .
\end{aligned}
$$

This holds for all $i$, and if we use $\rho_{R}^{\mathrm{GP}}\left(x_{i}\right) \leq \rho_{\alpha, \max }$ in (4.14), we get

$$
Q_{\alpha}(f) \geq \frac{\rho_{\alpha, \min }}{\rho_{\alpha, \max }} E^{\mathrm{hom}}\left(n_{\alpha}, L\right)-8 \pi a \rho_{\alpha, \max } n_{\alpha}
$$

where $E^{\text {hom }}$ is the energy in a box without an external potential.

Remark: If we had not taken Step 2 and used instead the division into boxes directly on the original Hamiltonian (1.1) we would be considering the minimization of

$$
\sum_{\alpha} E^{\mathrm{hom}}\left(n_{\alpha}, L\right)+V_{\alpha, \min } n_{\alpha}
$$


Such a procedure, however, would not lead to the GP energy. To see this, consider the special case of no interaction, i.e., $v=0$ and hence also also $E^{\text {hom }}\left(n_{\alpha}, L\right)=0$. The minimum of (4.18) is then simply $N \min _{x} V(x)$, whereas the GP energy is in this case $N$ times the ground state energy of $-\nabla^{2}+V$.

Step 4 (Minimizing in each box): Dropping the subsidiary condition $\sum n_{\alpha}=N$ can only lower the infimum. Hence it is sufficient to minimize each $Q_{\alpha}$ separately. To justify the use of (4.1), we have to ensure that $n_{\alpha}$ is large enough. But if the minimum is taken for some $\bar{n}_{\alpha}$, we have

$$
\frac{\rho_{\alpha, \min }}{\rho_{\alpha, \max }}\left(E^{\mathrm{hom}}\left(\bar{n}_{\alpha}+1, L\right)-E^{\mathrm{hom}}\left(\bar{n}_{\alpha}, L\right)\right) \geq 8 \pi a \rho_{\alpha, \max },
$$

and using Theorem 3.2, which states that

$$
E^{\mathrm{hom}}\left(\bar{n}_{\alpha}+1, L\right)-E^{\mathrm{hom}}\left(\bar{n}_{\alpha}, L\right) \leq 8 \pi a \frac{\bar{n}_{\alpha}}{L^{3}}\left(1+O\left(\bar{n}_{\alpha} a^{3} / L^{3}\right)\right),
$$

we see that $\bar{n}_{\alpha}$ is at least $\sim \rho_{\alpha, \max } L^{3}$. We shall later choose $L \sim N^{-1 / 10}$, so the conditions needed for (4.1) are fulfilled for $N$ large enough, since $\rho_{\alpha, \max } \sim N$ and hence $\bar{n}_{\alpha} \sim N^{7 / 10}, L / a \sim N^{9 / 10}$ and $Y_{\alpha} \sim N^{-2}$. Thus we have (for large enough $N$ )

$$
Q_{\alpha}(f) \geq 4 \pi a\left(\frac{\rho_{\alpha, \min }}{\rho_{\alpha, \max }} \frac{n_{\alpha}^{2}}{L^{3}}\left(1-C Y_{\alpha}^{1 / 17}\right)-2 n_{\alpha} \rho_{\alpha, \max }\right) .
$$

We now use $Y_{\alpha}=a^{3} n_{\alpha} / L^{3} \leq a^{3} N / L^{3} \equiv Y$, and drop the requirement that $n_{\alpha}$ has to be an integer. The minimum of (4.21) is obtained for

$$
n_{\alpha}=\frac{\rho_{\alpha, \max }^{2}}{\rho_{\alpha, \min }} \frac{L^{3}}{\left(1-C Y^{1 / 17}\right)} \text {. }
$$

This gives for (4.11)

$$
\begin{aligned}
& E^{\mathrm{QM}}(N, a)-E_{R}^{\mathrm{GP}}(N, a) \geq \\
& 4 \pi a \bar{\rho}_{R} N-4 \pi a \sum_{\alpha} \rho_{\alpha, \min }^{2} L^{3}\left(\frac{\rho_{\alpha, \max }^{3}}{\rho_{\alpha, \text { min }}^{3}} \frac{1}{\left(1-C Y^{1 / 17}\right)}\right) .
\end{aligned}
$$

Now $\rho_{R}^{\text {GP }}$ is differentiable by Lemma A.6, and strictly positive. Since all the boxes are in the fixed cube $\Lambda_{R}$ there are constants $C^{\prime}<\infty, C^{\prime \prime}>0$, such that

$$
\rho_{\alpha, \max }-\rho_{\alpha, \min } \leq N C^{\prime} L, \quad \rho_{\alpha, \min } \geq N C^{\prime \prime}
$$


Therefore we have, for $Y$ and $L$ small,

$$
\frac{\rho_{\alpha, \max }^{3}}{\rho_{\alpha, \min }^{3}} \frac{1}{\left(1-C Y^{1 / 17}\right)} \leq 1+D Y^{1 / 17}+D^{\prime} L
$$

with suitable constants $D$ and $D^{\prime}$. Also,

$$
4 \pi a \sum_{\alpha} \rho_{\alpha, \min }^{2} L^{3} \leq 4 \pi a \int\left(\rho_{R}^{\mathrm{GP}}\right)^{2} \leq E_{R}^{\mathrm{GP}}(N, a),
$$

and hence

$$
E^{\mathrm{QM}}(N, a) \geq E_{R}^{\mathrm{GP}}(N, a)\left(1-D Y^{1 / 17}-D^{\prime} L\right) .
$$

As last step it remains to optimize the length $L$. Recall that $Y=a^{3} N / L^{3}$ and $N a$ is fixed. The exponents of $N$ in both error terms in (4.27) are the same for

$$
L \sim a N^{9 / 10} \sim N^{-1 / 10}
$$

The final result, therefore, is

$$
E^{\mathrm{QM}}(N, a) \geq E_{R}^{\mathrm{GP}}(N, a)\left(1-D^{\prime \prime} N^{-1 / 10}\right) .
$$

\subsection{The limit theorems}

By Theorems 3.1 and 4.1 we have (with $a=a_{1} / N$ )

$$
E^{\mathrm{GP}}(N, a)\left(1+O\left(N^{-2 / 3}\right)\right) \geq E^{\mathrm{QM}}(N, a) \geq E_{R}^{\mathrm{GP}}(N, a)\left(1-O\left(N^{-1 / 10}\right)\right) .
$$

Dividing by $N$ and taking the limit $N \rightarrow \infty$ we have

$$
E^{\mathrm{GP}}\left(1, a_{1}\right) \geq \lim _{N \rightarrow \infty} \frac{E^{\mathrm{QM}}\left(N, a_{1} / N\right)}{N} \geq E_{R}^{\mathrm{GP}}\left(1, a_{1}\right)
$$

for all $R$ large enough. Using Lemma 2.2 and taking the limit $R \rightarrow \infty$ we finally prove Theorem 1.1.

The convergence of the energy implies also the convergence of the densities: We replace $V$ by $V+\delta W$ with $W \in L^{\infty}$, and denote the corresponding 
energies by $E_{\delta}(N, a)$. It is no restriction to assume that $V+\delta W \geq 0$ for small $|\delta| . E_{\delta}^{\mathrm{QM}}\left(N, a_{1} / N\right) / N$ is concave in $\delta$ (it is an infimum over linear functions), and converges for each $\delta$ to $E_{\delta}^{\mathrm{GP}}\left(1, a_{1}\right)$ as $N \rightarrow \infty$. This implies convergence of the derivatives and we have (Feynman-Hellmann principle)

$$
\left.\frac{\partial}{\partial \delta} E_{\delta}^{\mathrm{QM}}(N, a)\right|_{\delta=0}=\int_{\mathbb{R}^{3}} W \rho_{N, a}^{\mathrm{QM}},\left.\quad \frac{\partial}{\partial \delta} E_{\delta}^{\mathrm{GP}}(N, a)\right|_{\delta=0}=\int_{\mathbb{R}^{3}} W \rho_{N, a}^{\mathrm{GP}}
$$

with $\rho_{N, a}^{\mathrm{QM}}$ given by (1.3). In the weak $L^{1}$ sense we thus have

$$
\lim _{N \rightarrow \infty} \frac{1}{N} \rho_{N, a_{1} / N}^{\mathrm{QM}}(x)=\rho_{1, a_{1}}^{\mathrm{GP}}(x),
$$

which proves Theorem 1.2 .

\section{Conclusions}

We have proved that the GP energy functional correctly describes the energy and particle density of a Bose gas in a trap to leading order in the small parameter $\bar{\rho} a^{3}$ (where $\bar{\rho}$ is the mean density and $a$ is the scattering length) in the limit where the particle number $N$ tends to infinity, but $a$ tends to zero with $\mathrm{Na}$ fixed.

\section{Appendix A}

In this appendix we prove Theorem 2.1. The proof is split into several lemmas.

Lemma A.1 (Strict convexity). For $\rho \geq 0, \sqrt{\rho} \in \mathcal{D}, \mathcal{E}^{\mathrm{GP}}[\sqrt{\rho}]$ is strictly convex in $\rho$.

Proof. The second term in (1.4) is linear, the third quadratic in $\rho$. So it suffices to show that the first term is convex. Let $\rho_{1}$ and $\rho_{2}$ be given, with $\Phi_{1}=\rho_{1}^{1 / 2}$ and $\Phi_{2}=\rho_{2}^{1 / 2}$ in $\mathcal{D}_{N}$. Then also $\Phi=\left(\alpha \rho_{1}+(1-\alpha) \rho_{2}\right)^{1 / 2} \in \mathcal{D}_{N}$ for all $0<\alpha<1$. We have

$$
\begin{aligned}
\Phi \nabla \Phi & =\alpha \Phi_{1} \nabla \Phi_{1}+(1-\alpha) \Phi_{2} \nabla \Phi_{2} \\
& =\left(\alpha^{1 / 2} \Phi_{1}\right)\left(\alpha^{1 / 2} \nabla \Phi_{1}\right)+\left((1-\alpha)^{1 / 2} \Phi_{2}\right)\left((1-\alpha)^{1 / 2} \nabla \Phi_{2}\right) \\
& \leq\left(\alpha \Phi_{1}^{2}+(1-\alpha) \Phi_{2}^{2}\right)^{1 / 2}\left(\alpha\left|\nabla \Phi_{1}\right|^{2}+(1-\alpha)\left|\nabla \Phi_{2}\right|^{2}\right)^{1 / 2} \\
& =\Phi\left(\alpha\left|\nabla \Phi_{1}\right|^{2}+(1-\alpha)\left|\nabla \Phi_{2}\right|^{2}\right)^{1 / 2} .
\end{aligned}
$$


Hence

$$
|\nabla \Phi|^{2} \leq \alpha\left|\nabla \Phi_{1}\right|^{2}+(1-\alpha)\left|\nabla \Phi_{2}\right|^{2}
$$

Remark. Because $V \geq 0, \mathcal{E}^{\mathrm{GP}}[\Phi]$ is also convex in $\Phi \in \mathcal{D}$. But since the domain $\mathcal{D}_{N}$ is not convex, it is necessary to consider $\rho \mapsto \mathcal{E}^{\mathrm{GP}}[\sqrt{\rho}]$.

Lemma A.2 (Minimizer). For all $N$ there exists a minimizing $\Phi_{\infty} \in \mathcal{D}_{N}$, with $\mathcal{E}^{\mathrm{GP}}\left[\Phi_{\infty}\right]=E^{\mathrm{GP}}(N, a)$. Moreover, $\left|\Phi_{\infty}\right|^{2}$ is unique.

Proof. Let $\Phi_{n}$ be a minimizing sequence in $\mathcal{D}_{N}$, i.e., $\lim _{n \rightarrow \infty} \mathcal{E}^{\mathrm{GP}}\left[\Phi_{n}\right]=E^{\mathrm{GP}}$. It is clear that there exists a constant $C$, such that $\left\|\nabla \Phi_{n}\right\|_{2}<C,\left\|\Phi_{n}\right\|_{4}<$ $C$ and $\int\left|\Phi_{n}\right|^{2} V<C$ for all $n$ (recall that $V$ is nonnegative). Hence the sequence belongs to a weakly compact set in $L^{4}$, as well as in the Sobolev space $H^{1}=\left\{\Phi:\|\Phi\|_{2}^{2}+\|\nabla \Phi\|_{2}^{2}<\infty\right\}$, and in the space $L_{V}^{2}$, defined by the $L^{2}$ norm $\|\Phi\|_{V}=\left(\int|\Phi(x)|^{2} V(x) d^{3} x\right)^{1 / 2}$. Thus, there exists a $\Phi_{\infty} \in \mathcal{D}$ and a weakly convergent subsequence, again denoted by $\Phi_{n}$, such that

$$
\begin{gathered}
\Phi_{n} \rightarrow \Phi_{\infty} \text { in } L^{2} \cap L^{4} \cap L_{V}^{2} \\
\nabla \Phi_{n} \rightarrow \nabla \Phi_{\infty} \text { in } L^{2} .
\end{gathered}
$$

Because the $L^{4}$ norm, the Sobolev norm, and the $L_{V}^{2}$ norm are all weakly lower semicontinuous, we have

$$
\liminf _{n \rightarrow \infty} \mathcal{E}^{\mathrm{GP}}\left[\Phi_{n}\right] \geq \mathcal{E}^{\mathrm{GP}}\left[\Phi_{\infty}\right]
$$

and it remains only to show that $\Phi_{\infty} \in \mathcal{D}_{N}$. Since $\left|\Phi_{n}\right|^{2}$ converges to $\left|\Phi_{\infty}\right|^{2}$ in $L_{\text {loc }}^{1}$ it is clear that $\left\|\Phi_{\infty}\right\|_{2}^{2} \leq N$. Moreover,

$$
\int_{B}\left|\Phi_{n}\right|^{2} \stackrel{n \rightarrow \infty}{\longrightarrow} \int_{B}\left|\Phi_{\infty}\right|^{2} \leq\left\|\Phi_{\infty}\right\|_{2}^{2}
$$

for all bounded regions $B$. If $\left\|\Phi_{\infty}\right\|_{2}^{2}=N-\epsilon$ with $\epsilon>0$, then there exists a constant $M_{B}$ for all $B$, such that

$$
\int_{\mathbb{R}^{3} \backslash B}\left|\Phi_{n}\right|^{2} \geq \epsilon
$$

for all $n>M_{B}$. Since $\lim _{|x| \rightarrow \infty} V(x)=\infty$, this would imply $\int V\left|\Phi_{n}\right|^{2} \rightarrow \infty$, which is impossible because $\Phi_{n}$ is a minimizing sequence for the functional $\mathcal{E}^{\mathrm{GP}}$. Hence $\left\|\Phi_{\infty}\right\|_{2}^{2}=N$.

The uniqueness of $\left|\Phi_{\infty}\right|^{2}$ follows immediately from strict convexity, Lemma A.1. 
Lemma A.3 (GP equation). Every minimizing $\Phi_{\infty}$ satisfies the GrossPitaevskii equation (2.4). Conversely, every solution to (2.4), with $\mu$ given by (2.5), is a minimizer for $\mathcal{E}^{\mathrm{GP}}$.

Proof. Pick a function $f \in C_{0}^{\infty}$. The stationarity of $\mathcal{E}^{\mathrm{GP}}$ at $\Phi_{\infty}$ implies

$$
\left.\frac{\partial}{\partial \epsilon}\left(\mathcal{E}^{\mathrm{GP}}\left[\Phi_{\infty}+\epsilon f\right]+\mu\left\|\Phi_{\infty}+\epsilon f\right\|_{2}^{2}\right)\right|_{\epsilon=0}=0
$$

with a Lagrange parameter $\mu$ to take account of the subsidiary condition $\|\Phi\|_{2}^{2}=N$. With $f$ real valued one obtains

$$
-\nabla^{2} \operatorname{Re} \Phi_{\infty}+V \operatorname{Re} \Phi_{\infty}+8 \pi a\left|\Phi_{\infty}\right|^{2} \operatorname{Re} \Phi_{\infty}=\mu \operatorname{Re} \Phi_{\infty}
$$

and an analogous equation for $\operatorname{Im} \Phi_{\infty}$ with $f$ purely imaginary. The value of $\mu$ is obtained by multiplying the GP equation with $\Phi_{\infty}$ and integrating. By the same argument, every solution $\Phi$ to the GP equation satisfies $\mathcal{E}^{\mathrm{GP}}[\Phi]=E^{\mathrm{GP}}$ and is thus a minimizer.

Lemma A.4 (Uniqueness). The minimizing $\Phi_{\infty}$ is unique up to a constant phase factor. This factor can be chosen so that $\Phi_{\infty}$ is strictly positive.

Proof. Since $\mathcal{E}^{\mathrm{GP}}[|\Phi|] \leq \mathcal{E}^{\mathrm{GP}}[\Phi]$ (by an analogous computation as in the proof of Lemma A.1), we know that $\left|\Phi_{\infty}\right|$ is a minimizer and hence a solution to the GP equation. It is thus an eigenstate of the Hamiltonian $H=-\nabla^{2}+W$ with $W=V+8 \pi a\left|\Phi_{\infty}\right|^{2}$ (recall that $\left|\Phi_{\infty}\right|^{2}$ is unique), and since it is nonnegative, it must be a ground state. Since $\Phi_{\infty}$ solves the same equation it is also a ground state. Now $W \in L_{\text {loc }}^{2}$ and $\lim _{|x| \rightarrow \infty} W(x)=\infty$, so the ground state of $H$ is unique up to a phase and without zeros (see [7], XIII.47).

The unique strictly positive minimizer is denoted by $\Phi^{\mathrm{GP}}$.

Lemma A.5 (Exponential fall-off). For all $t>0$ there exists an $M_{t}$, such that $\Phi^{\mathrm{GP}}(x) \leq M_{t} e^{-t|x|}$. In particular, $\Phi^{\mathrm{GP}} \in L^{\infty}$.

Proof. Put $W=V+8 \pi a\left(\Phi^{\mathrm{GP}}\right)^{2}$ and let $t>0$. The GP equation implies

$$
\left(-\nabla^{2}+t^{2}\right) \Phi^{\mathrm{GP}}=-\left(W-\mu-t^{2}\right) \Phi^{\mathrm{GP}} .
$$


Using the Yukawa potential $Y_{t}(x)=(4 \pi|x|)^{-1} \exp (-t|x|)$ we can rewrite this as

$$
\Phi^{\mathrm{GP}}(x)=-\int Y_{t}(x-y)\left(W(y)-\mu-t^{2}\right) \Phi^{\mathrm{GP}}(y) d^{3} y
$$

Since $\Phi^{\mathrm{GP}}>0$, and $W(y)-\mu-t^{2}>0$ for $|y|>R$ with $R$ large enough, we also have

$$
\Phi^{\mathrm{GP}}(x) \leq-\int_{|y|<R} Y_{t}(x-y)\left(W(y)-\mu-t^{2}\right) \Phi^{\mathrm{GP}}(y) d^{3} y .
$$

Now $W \Phi^{\mathrm{GP}} \in L_{\mathrm{loc}}^{2}$, and hence

$$
M_{t}=\sup _{x} \int_{|y|<R} \frac{\exp \{t(|x|-|x-y|)\}}{4 \pi|x-y|}\left(W(y)-\mu-t^{2}\right) \Phi^{\mathrm{GP}}(y) d^{3} y<\infty .
$$

Lemma A.6 (Regularity). $\Phi^{\mathrm{GP}}(x)$ is once continuously differentiable in $x \in \mathbb{R}^{3}$, and $\nabla \Phi^{\mathrm{GP}}$ is Hölder continuous of order 1 . If $V \in C^{\infty}$, then $\Phi^{\mathrm{GP}} \in C^{\infty}$. Moreover, $E^{\mathrm{GP}}(N, a)$ is continuously differentiable in a and hence in $N$ (by Eq. (1.6)), and $d E^{\mathrm{GP}}(N, a) / d N$ satisfies (2.5).

Proof. The last lemma and the GP equation imply $\nabla^{2} \Phi^{\mathrm{GP}} \in L_{\text {loc }}^{\infty}$. Thus $\nabla \Phi^{\mathrm{GP}}$ exists and is Hölder continuous (see [9], 10.2). The $C^{\infty}$ property follows by a bootstrap argument. The differentiability with respect to the parameter a may be shown by a Feynman-Hellmann type argument like analogous statements (e.g. differentiability w.r.t. nuclear charges) in TF theory [8]. Eq. (2.5) follows immediately from (1.6) and $E^{\mathrm{GP}}(1, N a)=\mathcal{E}^{\mathrm{GP}}\left[\Phi_{1, N a}^{\mathrm{GP}}\right]$.

Lemmas A.1 A.6 complete the proof of Theorem 2.1.

\section{Appendix B}

In this appendix we show that (4.1) holds for nonnegative potentials satisfying (4.2), and that a similar estimate with $1 / 17$ replaced by $O(\varepsilon)$ holds under the condition (4.3). 
We cut the potential at a finite radius $\widetilde{R}$ which, because of $v \geq 0$, can only decrease the energy. We thus define

$$
\widetilde{v}(r)=v(r) \Theta(\widetilde{R}-r)
$$

and denote the corresponding scattering length by $\widetilde{a} \leq a$. Let $u$ be the zero energy scattering solution for the potential $v$ (cf. (1.2)) and put

$$
h(r)=r-\frac{u(r)}{u^{\prime}(r)} .
$$

The difference $a-\widetilde{a}$ can be estimated as follows. Since $v(r)$ and $\widetilde{v}(r)$ agree for $r \leq \widetilde{R}$, the same holds for the corresponding scattering solutions. Moreover, $\widetilde{a}=h(\widetilde{R})$. Hence

$$
\begin{aligned}
a-\widetilde{a} & =\int_{\widetilde{R}}^{\infty} h^{\prime}(r) d r=\int_{\widetilde{R}}^{\infty} \frac{u(r) u^{\prime \prime}(r)}{u^{\prime}(r)^{2}} d r \\
& \leq \int_{\widetilde{R}}^{\infty} \frac{u^{\prime \prime}(r)}{u(r)} r^{2} d r=\frac{1}{2} \int_{\widetilde{R}}^{\infty} v(r) r^{2} d r
\end{aligned}
$$

where convexity of $u$ has been used to derive the inequality. We remark that for $\widetilde{R} \rightarrow 0$ this simple estimate gives the Spruch-Rosenberg inequality 12

$$
a \leq \frac{1}{2} \int_{0}^{\infty} v(r) r^{2} d r
$$

Assuming (4.2) one obtains

$$
\widetilde{a} \geq a\left(1-\text { const. }\left(\frac{a}{\widetilde{R}}\right)^{\frac{1}{5}+\epsilon}\right) .
$$

Eq. (4.1) holds in any case with a replaced by $\widetilde{a}$, and if we we take $\widetilde{R} \propto a Y^{-5 / 17+\epsilon^{\prime}}$ with $\epsilon^{\prime}>0$ then the error in $(\mathrm{B} .5)$ is of higher order than the leading error term in (4.1). We have thus established (4.1) under the condition (4.2). If only the weaker condition (4.3) holds, then the additional error term may be $O\left(Y^{\left(5 / 17-\epsilon^{\prime}\right) \epsilon}\right)$. 
To see the significance of condition (4.3) we also estimate $a-\widetilde{a}$ from below:

$$
a-\widetilde{a} \geq \int_{\widetilde{R}}^{\infty} u(r) u^{\prime \prime}(r) \geq \frac{1}{2} \int_{\max (\widetilde{R}, a)}^{\infty} v(r)(r-a)^{2} d r
$$

In order that $a$ is finite the last integral must converge, i.e., a slower decrease than $1 / r^{3}$ is not allowed.

\section{References}

[1] E.P. Gross, Structure of a Quantized Vortex in Boson Systems, Nuovo Cimento 20, 454-466 (1961)

[2] L.P. Pitaevskii, Vortex lines in an imperfect Bose gas, Sov. Phys. JETP, 13, 451-454 (1961)

[3] E.P. Gross, Hydrodynamics of a superfluid condensate, J. Math. Phys. 4, 195-207 (1963)

[4] F. Dalfovo, S. Giorgini, L.P. Pitaevskii, S. Stringari, Theory of BoseEinstein condensation in trapped gases, Rev. Mod. Phys. 71, 463-512 (1999)

[5] E.H. Lieb, J. Yngvason, Ground State Energy of the low density Bose Gas, Phys. Rev. Lett. 80, 2504-2507 (1998)

[6] F.J. Dyson, Ground-State Energy of a Hard-Sphere Gas, Phys. Rev. 106, 20-24 (1957)

[7] M. Reed, B. Simon, Methods of Modern Mathematical Physics IV, Academic Press, 1978

[8] E.H. Lieb, Thomas-fermi and related theories of atoms and molecules, Rev. Mod. Phys. 53, 603-641 (1981)

[9] E.H. Lieb, M. Loss, Analysis, Amer. Math. Society, 1997 
[10] E.H. Lieb, Simplified Approach to the Ground State Energy of an Imperfect Bose Gas, Phys. Rev. 130, 2518-2528 (1963). See also Phys. Rev. 133, A899-A906 (1964) (with A.Y. Sakakura) and Phys. Rev. 134, A312-A315 (1964) (with W. Liniger).

[11] P.L. Lions, Two Geometrical Properties of Solutions of Semilinear Problems, Applicable Analysis, Vol. 12, 267-272 (1981)

[12] L. Spruch, L. Rosenberg, Upper bounds on Scattering Lengths for Static Potentials, Phys. Rev. 116, 1034 (1959) 